\title{
Occult Acute Macular Neuroretinopathy
}

\author{
Michael S. Vaphiades and Brendan Grondines \\ Department of Ophthalmology, University of Alabama, Birmingham, Alabama, USA
}

\begin{abstract}
A 19-year-old Caucasian woman developed an upper respiratory infection, took a cold formulation containing $5 \mathrm{mg}$ of phenylephrine, and developed a very rare and unusual form of acute macular neuroretinopathy (AMN) that could not be detected on fundoscopic examination, visual fields, nor electrophysiological testing. Spectral-domain optical coherence tomography (SD-OCT) revealed a lesion limited to the fovea. This case illustrates the value of SD-OCT, in light of otherwise normal testing, in a variant of AMN the authors call "occult AMN".
\end{abstract}

\section{ARTICLE HISTORY}

Received 2 February 2017

Accepted 18 February 2017

\section{KEYWORDS}

Acute macular

neuroretinopathy;

phenylephrine;

spectral-domain optical

coherence tomography

\section{Introduction}

Acute macular neuroretinopathy (AMN) is a rare retinal disorder of unknown pathogenesis defined by the presence of foveal reddish-brown, "flower petal"-shaped lesions that correspond to impaired visual acuity and central/paracentral scotomas on visual field testing. Advances in multimodal imaging, namely, spectral-domain optical coherence tomography (SD-OCT), have led to better anatomical characterisation and understanding of this of this rare retinal disorder.

\section{Case presentation}

A 19-year-old Caucasian woman developed an upper respiratory infection and took a cold formulation containing $5 \mathrm{mg}$ of phenylephrine. She took 2 pills daily for 3 consecutive days. She then noted a small central scotoma in the right eye (OD) on the second day. She was otherwise healthy with no past medical history and on no other medications. She was evaluated in the neuroophthalmology clinic 5 days later. There was a suspicion of acute macular neuroretinopathy (AMN) because of the history of phenylephrine exposure. Her neuro-ophthalmologic examination included a normal blood pressure and heart rate, a best-corrected visual acuity of 20/40 in both eyes (OU), normal colour vision OU, and normal pupillary function. There was a small central defect on an Amsler grid OD that she did not appreciate in the left eye (OS); however, an automated visual field was normal OU (Figure 1). Her ocular motility and slit-lamp examinations were normal. Most importantly, the fundus was completely normal, without macular abnormality, and lacking the usual "flower petal" appearance of AMN (Figure 2). The efferent examination was normal. Other testing included a normal visual evoked potential (VEP) and multifocal electroretinogram (mfERG) (Figure 3). Spectral-domain optical coherence tomography (SD-OCT) showed a hyper-reflective lesion in the outer nuclear layer, photoreceptor layer, and retinal pigment epithelium (RPE) OU (Figure 4). She was evaluated 1 week later with improvement of the SD-OCT (Figure 5) and a best-corrected acuity of 20/ 25 OU.

\section{Discussion}

Acute macular neuroretinopathy (AMN) is a rare retinal disorder first reported by Bos and Deutman in 1975. They described four young women who presented with acute unilateral or bilateral paracentral visual loss accompanied by a brownish-reddish wedge-shaped macular petaloid configuration pointing to the fovea. ${ }^{1}$ In 2003, Turbeville and colleagues

CONTACT Michael S. Vaphiades, DO mvaphiades@uabmc.edu Callahan Eye Hospital, Department of Ophthalmology University of Alabama, Suite 601,700 South 18th Street, Birmingham, AL 35233, USA.

Color versions of one or more of the figures in the article can be found online at www.tandfonline.com/ioph.

(c) 2017 Taylor \& Francis 


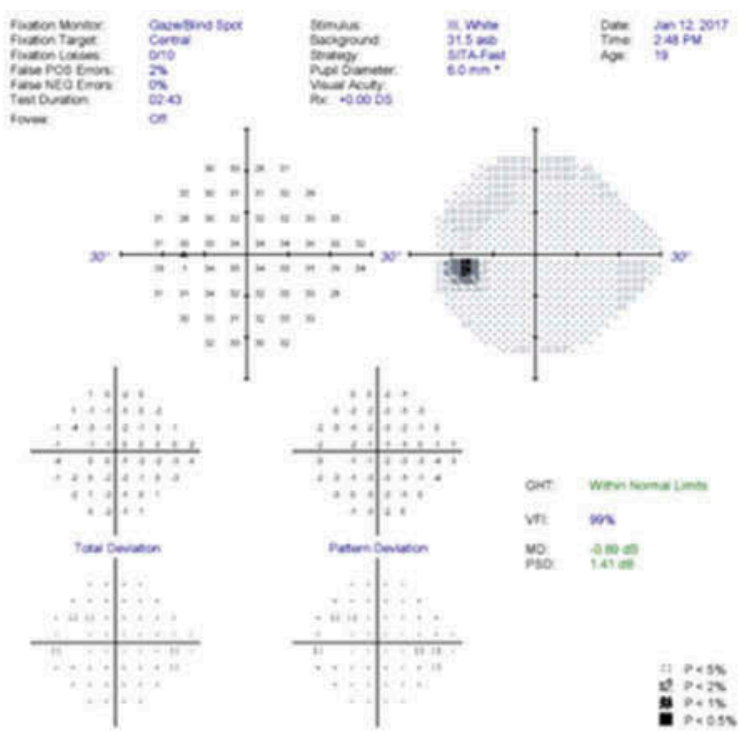

Left eye

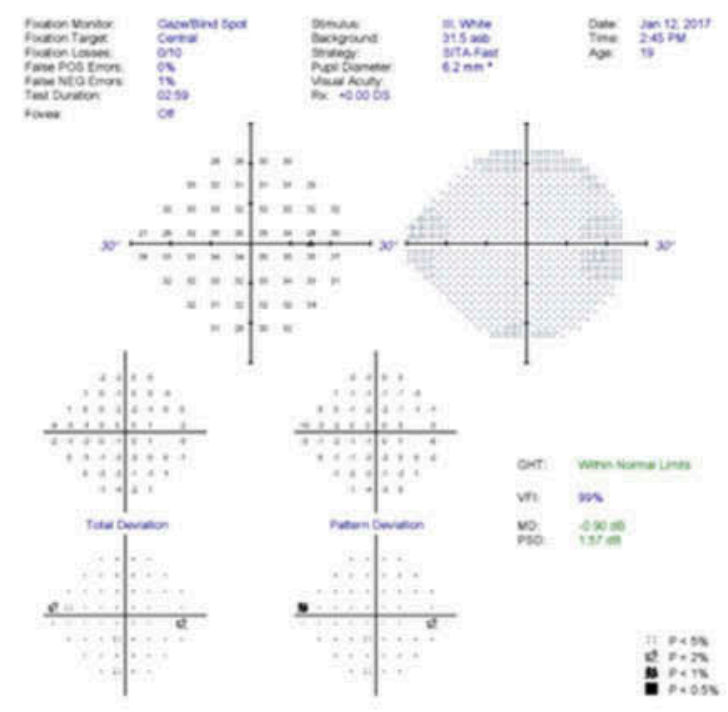

Right eye

Figure 1. Humphrey visual field SITA fast, 24-2, showing normal field OU.

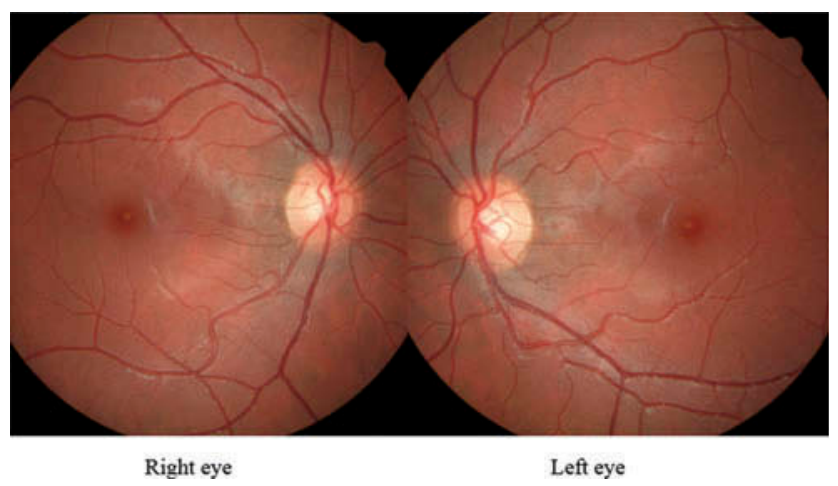

Figure 2. Digital fundus photographs of the right (left panel) and left (right panel) normal OU.

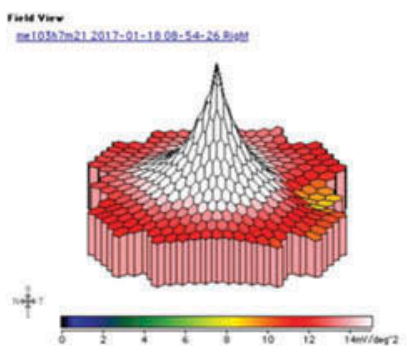

Right eye

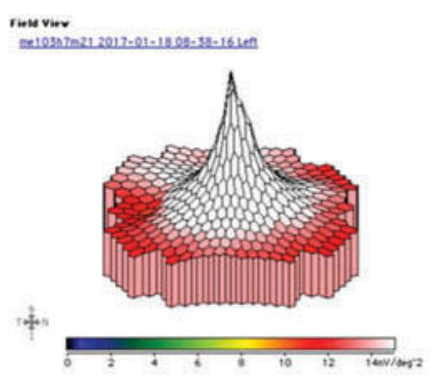

Left eye
Figure 3. Multifocal electroretinogram of the right eye (left panel) and left eye (right panel) normal OU.

analyzed 41 cases of AMN, 83\% of which were women in their reproductive years. Predisposing factors included oral contraception, antecedent upper respiratory/influenza-like symptoms, trauma, migraines, and hypotension. Intravenous fluorescein angiography (IVFA) and indocyanine green angiograms were normal in many of the cases expect for possible hypo-flourescence of some of the lesions. ${ }^{2}$ In 2016, Bhavsar and colleagues reported 101 cases identified from 1975 to December of 2014. They came to a similar conclusion: that AMN preferentially affects young, white women and over half in the third decade of life. It was most commonly associated with nonspecific flu-like illness, fever, or oral contraceptive use. ${ }^{3}$ The use of epinephrine or phenylephrine, common nasal decongestants, has been theorised as a potential causative agent of AMN. $^{4-7}$ In addition, a review of 902 cases reported by the US Federal Drug Administration's adverse events reporting system regarding epinephrine from 1980 to 1997 disclosed 3 cases that were listed as "retinal disease" or "vision abnormality". 2

From a diagnostic standpoint, mfERG has shown diminished amplitudes in AMN, the effects of which are presumably localised to the outer retina or at the level of the photoreceptor cells; however, normal findings have also been reported. ${ }^{3,8-11}$

AMN was originally described as an inner retinal disease based on clinical observations alone; however, when Feigl and Haas first reported optical coherence tomography (OCT) as a diagnostic modality in AMN, they found an area of hyper- 


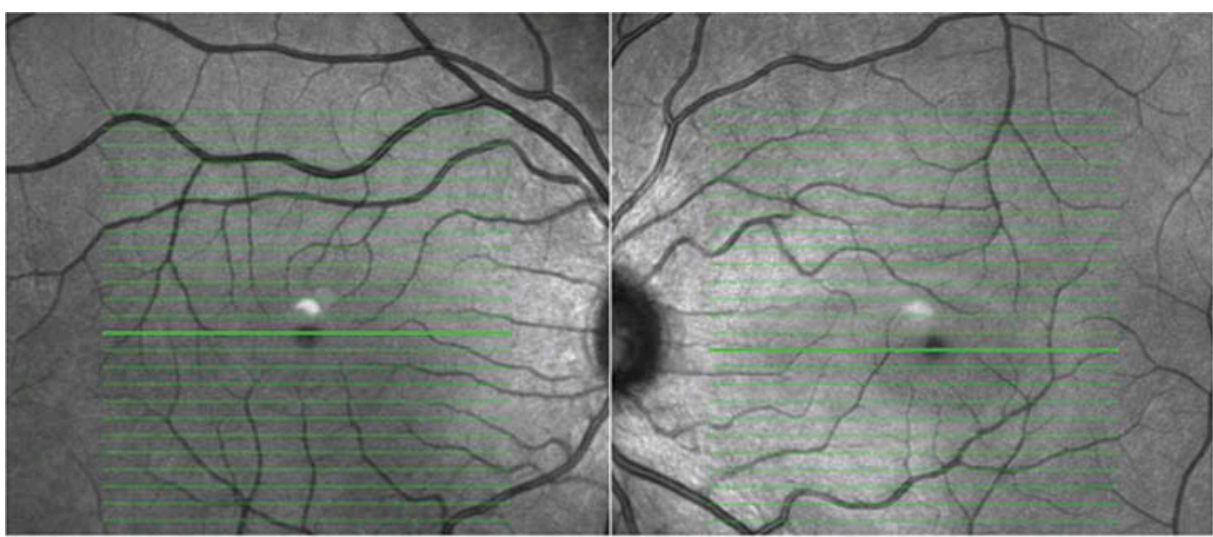

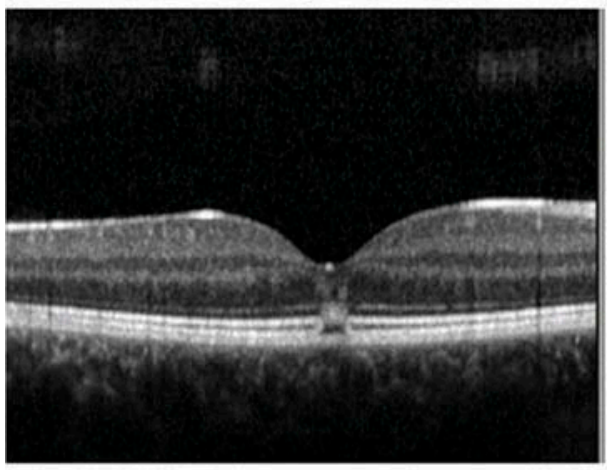

Right eye

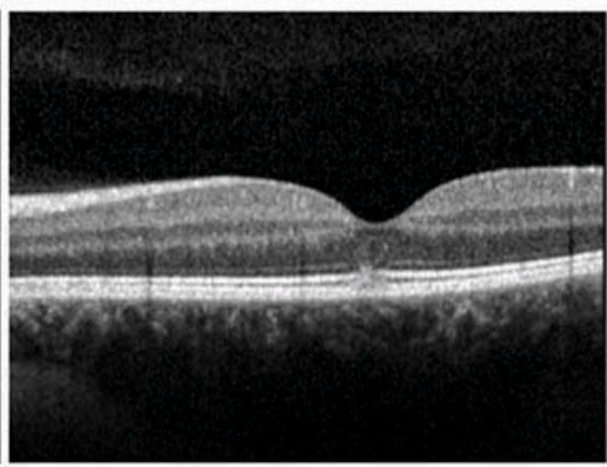

Left eye

Figure 4. Initial spectral-domain optical coherence tomography (OCT) showing an abnormal appearing outer retinal layer OU. The right eye (left panel) shows a hyper-reflective sub-foveal lesion extending from the external limiting membrane through the ellipsoid zone. Beneath the lesion, there is a hypo-reflective cavity that disrupts the superficial aspect of the RPE. Above the sub-foveal lesion there are two moderately reflective vertical bands in the outer nuclear layer extending from the outer plexiform layer to the subfoveal lesion. The left eye (right panel) shows a moderately reflective lesion that disrupts the myoid zone, ellipsoid zone, and RPE. There is also very subtle trace reflectivity in the outer nuclear layer.

reflectivity above an unaffected retinal pigment epithelium-choriocapillaris complex in the outer retina. ${ }^{12}$ Monson and colleagues used ultra-highresolution OCT and noted focal thinning of the outer retina, possibly at the level of the photoreceptor cell outer segments with distortion of the inner segment/outer segment junction. ${ }^{13}$ With the advent of spectral-domain optical coherence tomography (SD-OCT), it was determined that AMN lesions typically show hyper-reflectivity at the junction between the outer plexiform layer (OPL) and outer nuclear layer (ONL) and are frequently associated with disruption of both the ellipsoid zone (EZ) and interdigitation zone (IZ). ${ }^{3}$ Furthermore, anatomical data have been able to support the critical role of deep capillary plexus (DCP) compromise in AMN. ${ }^{3,14}$

In 2013, Sarraf and colleagues reported a novel variant of AMN called paracentral acute middle maculopathy (PAMM). They hypothesised that transient ischaemia of the DCP results in a macular lesion beneath the OPL in AMN and that transient ischaemia of the intermediate capillary plexus (ICP) results in a macular lesion above the OPL in PAMM. The lesion in our case lies beneath the OPL, which would be classified as AMN rather than PAMM. ${ }^{15}$ The human foveola is the rod-free region of the central retina with maximum cone photoreceptor packing density. The absence of blood vessels and overlaying inner retinal tissue are thought to maximise the optical quality of the foveal pit by reducing light scattering. This central avascular region, known as the foveal avascular zone (FAZ), exhibits considerable individual variation in the size and shape. ${ }^{16}$ Our patient's lesion, at least by SD-OCT measurement, appeared to be limited to the FAZ. Thus, neither transient ischaemia of the ICP nor transient ischaemia of the DCP presents a satisfying mechanism. ${ }^{17}$ Although an exclusively sub-foveal lesion is an atypical presentation for AMN, it has been documented in the past by Makino and Tampo. ${ }^{18}$ Another atypical finding in our case was 

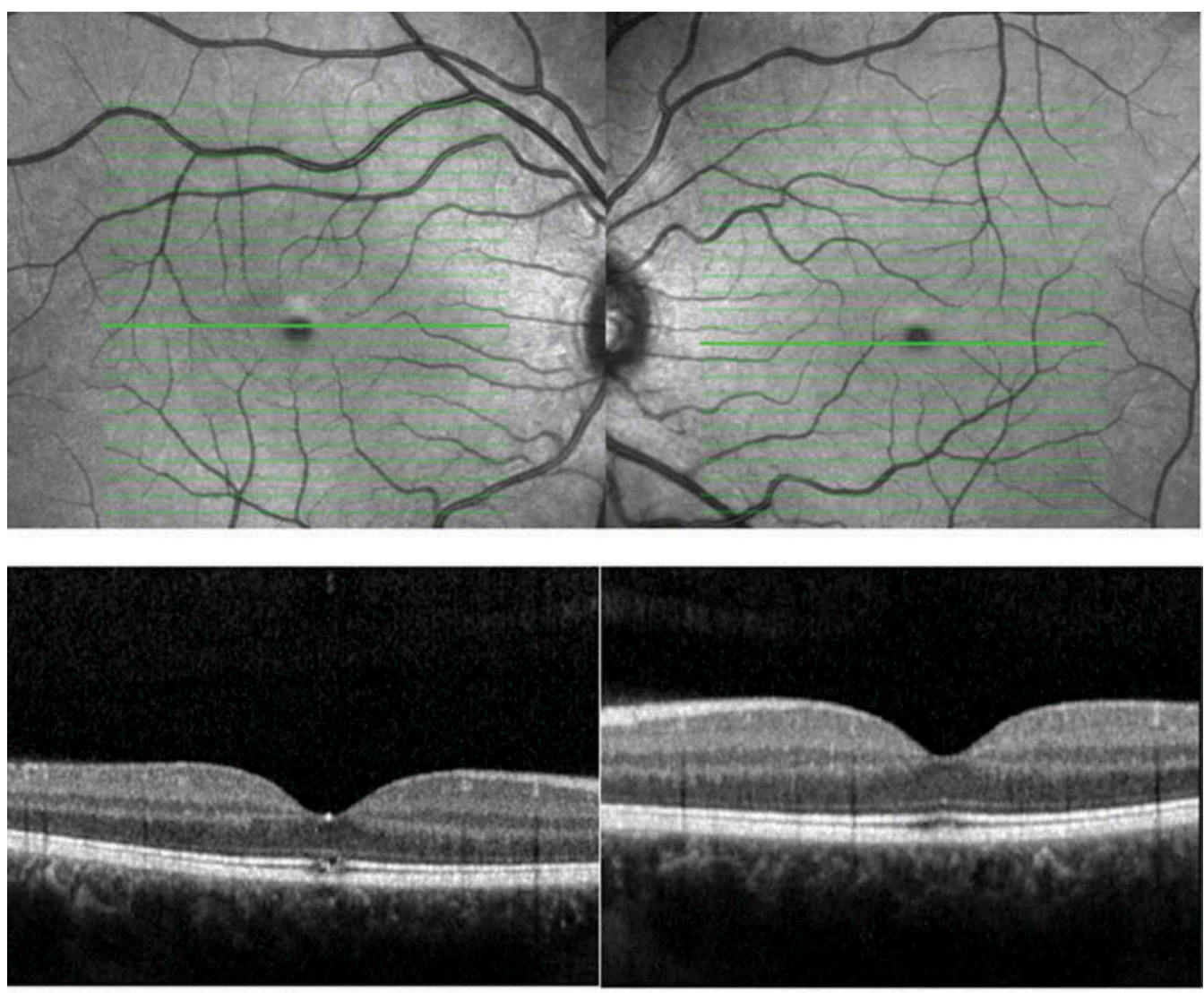

Right eye

Left eye

Figure 5. Subsequent (1 week later) spectral-domain optical coherence tomography (OCT) shows anatomical improvement of the outer retinal layers OU. The right eye (left panel) shows the sub-foveal lesion, still disrupting the ellipsoid zone; however, only the outline of the lesion is hyper-reflective, the centre is now hypo-reflective. There is also focal thinning of the RPE. The hyper-reflective vertical bands are no longer visible. The left eye (right panel) shows focal thinning of both the ellipsoid zone and RPE.

the normal fundoscopic examination. Even cases of early AMN generally show some evidence of retinal pathology on fundoscopy. ${ }^{19}$ Given both the normal fundoscopic examination as well as the presence of a lesion within the FAZ, our case could represent another variant of $A M N$, or perhaps suggest a nonischaemic pathogenesis for AMN.

In summary, AMN, even when it presents in the usual manner with decreased acuity, visual field defects, fundus findings, and electrophysiology and OCT abnormalities, is a rare and difficult diagnosis to render. Our patient could have very easily been labelled nonorganic or idiopathic, especially in light of the normal visual field and electrophysiological testing performed. Our patient was further difficult to diagnose because the macular lesion appears to be limited to only the fovea and not evident on fundoscopic examination, lacking the characteristic "flower petal" macular appearance that generally accompanies AMN. This case also illustrates the value of SD-OCT, in light of otherwise normal testing, in a variant of AMN we call "occult AMN."

\section{Declaration of interest}

The authors report no conflicts of interest. The authors alone are responsible for the content and writing of the article.

\section{Funding}

This work was supported in part by an unrestricted grant from the Research to Prevent Blindness, Inc., New York, New York, USA.

\section{References}

1. Bos PJ, Deutman AF. Acute macular neuroretinopathy. Am J Ophthalmol 1975;80:573-584. 
2. Turbeville SD, Cowan LD, Gass JD. Acute macular neuroretinopathy: a review of the literature. Surv Ophthalmol 2003;48:1-11.

3. Bhavsar KV, Lin S, Rahimy E, Joseph A, Freund KB, Sarraf D, Cunningham ET Jr. Acute macular neuroretinopathy: a comprehensive review of the literature. Surv Ophthalmol 2016;61:538-565.

4. O'Brien DM, Farmer SG, Kalina RE, Leon JA. Acute macular neuroretinopathy following intravenous sympathomimetics. Retina 1989;9:281-286.

5. Desai UR, Sudhamathi K, Natarajan S. Intravenous epinephrine and acute macular neuroretinopathy. Arch Ophthalmol 1993;111:1026-1027.

6. Guzak SV, Kalina RE, Chenoweth RG. Acute macular neuroretinopathy following adverse reaction to intravenous contrast media. Retina 1983;3:312-317.

7. El-Dairi M, Bhatti MT, Vaphiades MS. A shot of adrenaline. Surv Ophthalmol 2009;54:618-624.

8. Browning AC, Gupta R, Barber C, Lim CS, Amoaku WM. The multifocal electroretinogram in acute macular neuroretinopathy. Arch Ophthalmol 2003;121:1506-1507.

9. Maturi RK, Yu M, Sprunger DT. Multifocal electroretinographic evaluation of acute macular neuroretinopathy. Arch Ophthalmol 2003;121:1068-1069.

10. Chan WM, Liu DT, Tong JP, Law RW, Lam DS. Longitudinal findings of acute macular neuroretinopathy with multifocal electroretinogram and optical coherence tomography. Clin Exp Ophthalmol 2005;33:439-442.

11. Watzke RC, Shults WT. Annular macular neuroretinopathy and multifocal electroretinographic and optical coherence tomographic findings. Retina 2004;24:772775 .
12. Feigl B, Haas A. Optical coherence tomography (OCT) in acute macular neuroretinopathy. Acta Ophthalmol Scand 2000;78:714-716.

13. Monson BK, Greenberg PB, Greenberg E, Fujimoto JG, Srinivasan VJ, Duker JS. High-speed, ultra-high-resolution optical coherence tomography of acute macular neuroretinopathy. Br J Ophthalmol 2007;91:119-120.

14. Rahimy E, Sarraf D. Paracentral acute middle maculopathy spectral-domain optical coherence tomography feature of deep capillary ischemia. Curr Opin Ophthalmol 2014;25:207-212.

15. Sarraf D, Rahimy E, Fawzi AA, Sohn E, Barbazetto I, Zacks DN, Mittra RA, Klancnik JM Jr, Mrejen S, Goldberg NR, Beardsley R, Sorenson JA, Freund KB. Paracentral acute middle maculopathy: a new variant of acute macular neuroretinopathy associated with retinal capillary ischemia. JAMA Ophthalmol 2013;131:1275-1287.

16. Chui TY, Zhong Z, Song H, Burns SA. Foveal avascular zone and its relationship to foveal pit shape. Optom Vis Sci 2012;89:602-610.

17. Kim DY, Fingler J, Zawadzki RJ, Park SS, Morse LS, Schwartz DM, Fraser SE, Werner JS. Noninvasive imaging of the foveal avascular zone with high-speed, phase-variance optical coherence topography. Invest Ophthalmol Vis Sci 2012;53:85-92.

18. Makino S, Tampo H. Acute Macular Neuroretinopathy in a 15-year-old boy: optical coherence tomography and visual acuity findings. Case Rep Ophthalmol 2014;5:11-15.

19. Garg A, Shah AN, Richardson T, O'Sullivan E, Eleftheriadis H. Early features in acute macular neuroretinopathy. Int Ophthalmol 2014;34:685-688. 